\title{
REMARKS ON WIRE AND DIE BONDING FOR HYBRID CIRCUITS
}

\author{
A. SOFFA \\ Kulicke and Soffa Industries, Inc., Horsham, Pennsylvania 19044, USA
}

(Received June 8, 1977)

\begin{abstract}
Advances in hybrid automatic wire bonding include refined joy stick techniques, automatic alignment, a digitally operated head for higher speeds and flexibility, missing ball detection and programming aids. Advanced hybrid die attach equipment will have to accept a large variety of chip presentation techniques but will use CCTV and automatic alignment with microprocessor controls to aid programming and speed output.
\end{abstract}

\section{INTRODUCTION}

At the time of writing, automatic wire bonding of integrated circuits is maturing commercially, while the automatic wire bonding of hybrids is still in its early stages. Likewise, high speed die bonding of I.C.s is far in advance of automatic placement of semiconductor die in hybrid assembly. This paper will describe several advances that are presently in use, or are to be available shortly, that have particular importance when applied to hybrid wire and die bonding.

\section{MANUAL CORRECTION OF CHIP AND SUBSTRATE POSITION}

Several manual positioning or joystick devices are presently being used to move the substrate relative to the alignment crosshair. There is the 8-direction "onoff" switch type control; the joystick type which provides a table speed proportional to joystick displacement or pressure; and there are the displacement devices - either of the ball type to give motion simultaneously in the $\mathrm{X}-\mathrm{Y}$ directions or two separate rotary knobs, one for $\mathrm{X}$ and one for $\mathrm{Y}$, both of the displacement type.

Work done by Mehr \& Mehr ${ }^{1}$ of Measurement Systems Inc., of Norwalk, Connecticut, established by carefully controlled tests the superiority of the ball type displacement system rather than the "onoff" or speed proportional to pressure type. Our work substantiates this finding. We have also found little difference between the ball and the two separate XY displacement type control knobs, if the resistance to rotation is kept low. Apparently, the largest variable is in the reaction time for depressing the "enter" button. Experience has shown that first point in a 2-point entry type alignment takes approximately $2 \frac{1}{2}$ seconds and the second point entry about $1 \frac{1}{2}$ seconds.

\section{AUTOMATIC ALIGNMENT OF DIE}

Figure 1 is a curve useful in predicting output rates as a function of time per wire and alignment time. The curves are for 14-lead I.C.s with approximately $3 / 4$ second per I.C. allowed for all indexing and settling times. It shows that wire bonders, in the $.450 \mathrm{sec}$./ wire rate will yield approximately 325 units/hour with a 4 sec. operator-controlled alignment time. However, tests in the field with fully automatic alignment as associated with the K\&S Model 1414 Wire Bonder ${ }^{2}$ with average alignment times of approximately $1 / 2 \mathrm{sec}$. achieved rates of over 450 units/hour. Figure 2 is a plot of acquisition time data, including movement between two points and calculation time as a function of error of displacement. This data shows the advantage of having die placement errors of less than \pm 5 mils.

The automatic alignment system used in this work, see Figure 3, is of the pattern recognition type, where the "signature" of a portion of a die is obtained by scanning its projected image on the face of an image dissector tube. A half-mirror permits the die to be seen through a CCTV Camera and Monitor. A crosshair is provided at the image plane of the CCTV 


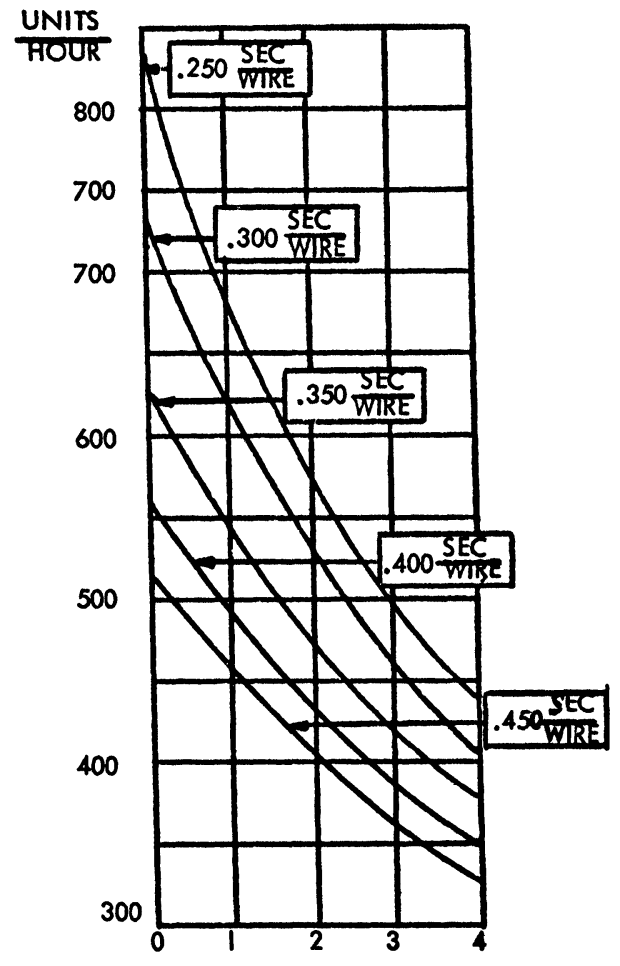

ALIGNMENT TIME (SECONDS)

FIGURE 114 lead I.C. production as function of alignment time.

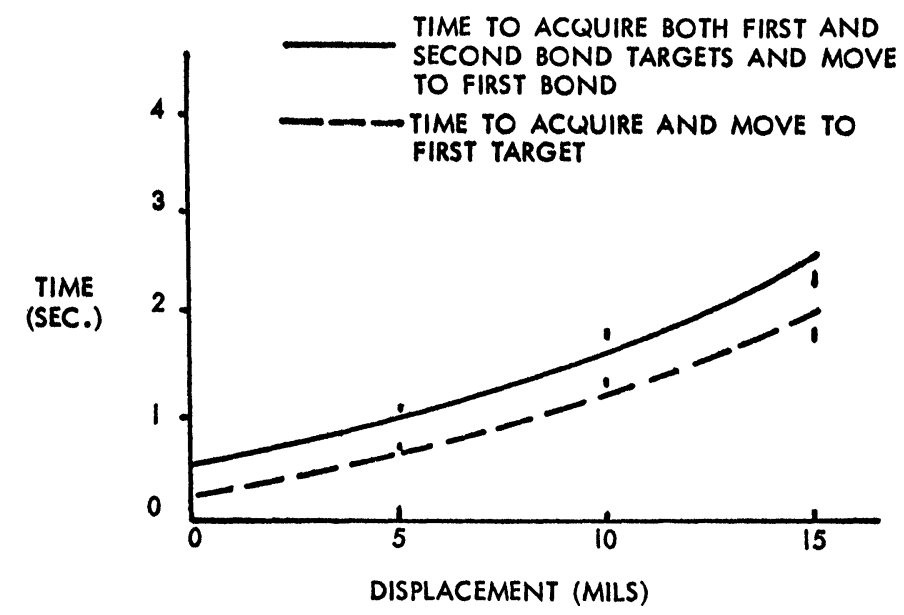

FIGURE 2 Time required for alignment of targets.

optical train for programming of the bond locations and tool position.

A circular scan is used such that variations in contrast are converted into a video signal which is further processed by filtering and digitizing. During programming, the signature of the annular zone of the die is stored in semiconductor memory as the reference signal. If the die is displaced in $\mathrm{X}, \mathrm{Y}$, or theta, the die is moved relative to the optical axis through a "search" mode, (either mechanically or electronically across the face of the image dissector tube) until the new video signal and the reference

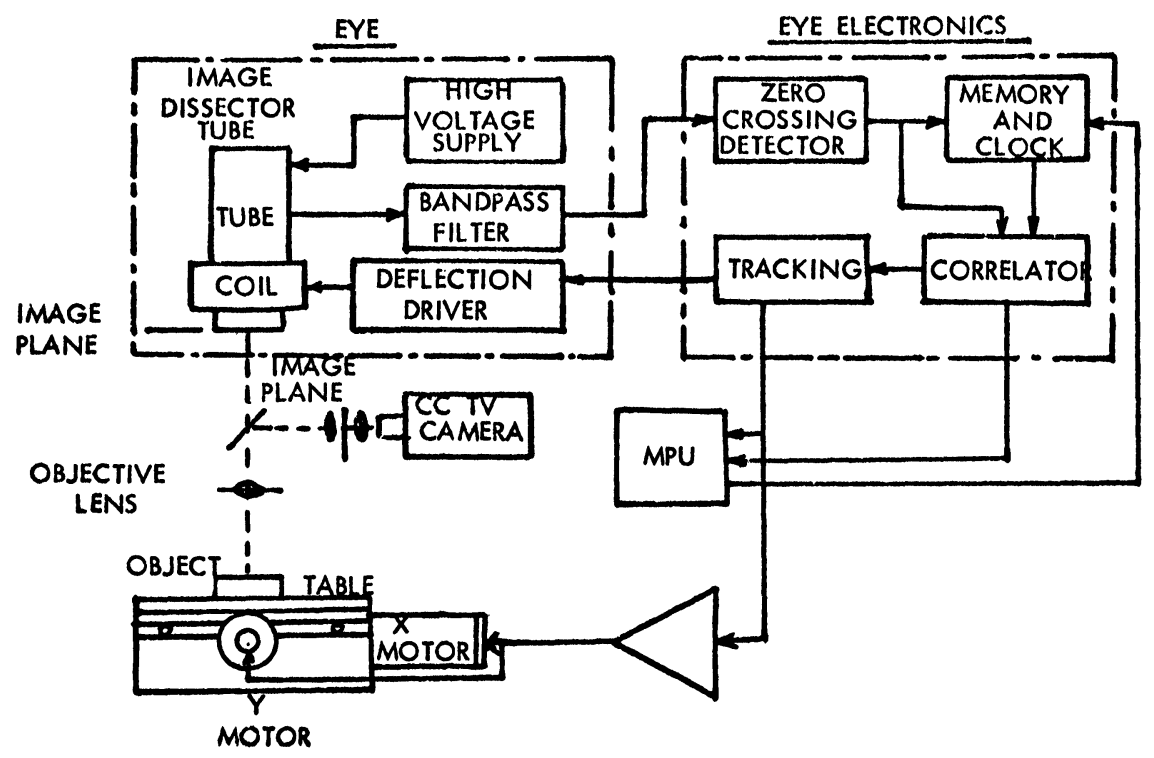

FIGURE 3 Schematics of automatic recognition system. 
signals are correlated. At this instant, a "tracking" mode takes over to allow the table servos to get their error signals from the $\mathrm{X}-\mathrm{Y}$-theta data provided by the correlator for final alignment. Displacements are in $\mathrm{X}, \mathrm{Y}$ and theta, but when a theta drive is not used, the theta is cancelled out electronically. After alignment, the coordinates of table position are recorded and the die is moved to a zone at a widely spread distance from the first point and this sequence of operation is then repeated. Two separate signatures are stored in memory for each chip and two addittional signatures for each different substrate.

\section{DIGITAL HEAD}

A digital ball bonding head is presently undergoing test at K\&S. This new design assures higher speeds per wire, fewer parts, compensation for bond heights, and offers complete flexibility of programming of such variables as bond time, bond force, loop height, loop tension control. These features are all highly desirable on hybrid work.

\section{COMPENSATION FOR VARIATIONS IN HEIGHT OF BOND ALIGNMENT SITES}

\begin{abstract}
Aligning targets for wire bonding on hybrids is made more severe by large variations in the height of the sites used for alignment. Normal variation on die thickness, the trend toward thicker wafers required by semiconductor manufacturers as the wafer diameters are increased, and normal variation in substrate thickness and waviness of substrates contribute to the problem. These problems are presently met with long depth of focus lens systems and by setting the focus sharply in the mean plane of the tops of the die and compromising with lesser resolution at the substrate level. Various other approaches, such as raising or lowering the work or zoom lenses and other optical compensation means are contemplated.
\end{abstract}

\section{PROGRAMMING AIDS}

Since comparatively large amounts of time are required for programming, various hardware and software features are available ${ }^{2-4}$ from the suppliers of machinery. In hardware there are battery supports to prevent loss of program, and tape or floppy discs for storage of the program. In software there is the ability to self-teach the program, the ability to use data from a computer aided design directly, the storage of die programs, easy correction of program, and easy substitution of entire die.

\section{MISSING BALL DETECTION}

When using fully automatic alignment, the wire bonder becomes an essentially unattended machine, thus a method of detecting failure to form a ball or other failures becomes paramount. At present, ${ }^{2}$ a missing ball detection system is used when a high voltage discharge method is used to form the ball. Here the failure to discharge the voltage at the appropriate point in the cycle adequately checks for failure to ballize.

\section{CRITERIA AND PROJECTED DESIGNS OF HYBRID DIE MOUNTERS}

The key requirement for a hybrid die attach system is flexibility. The hybrid manufacturer is usually not the semiconductor manufacturer and has the problem of accepting die with widely varying methods of die presentation. Substrates are just as varied. The most universally used method of supplying die is the waffle pack. The more gradations in size of pocket used by the supplier, the less time wasted during the mounting operation. Further care by the vendor in making sure each die is properly oriented in the respective pockets eliminates a source of trouble during assembly. Taxing the assembly operation with dies that are randomly oriented, can be offset if the hybrid manufacturer uses automatic alignment, or if the die can be re-pocketed or placed onto a tape ${ }^{5}$ or into a square spaced array.

Since the die, obviously, was in wafer array during probing and prior to separation, this is probably the most expeditious way of handling the die at hybrid die assembly.

If the hybrid manufacturer obtains the wafer in non-expanded form, he can then mount it onto a plastic member in non-expanded or expanded form. Both of these film-mounted die holding methods require plunge-up to facilitate their removal from the plastic film. Inked die can be by-passed during assembly, or a magnetic tape which contains the location of die of an acceptable quality can be synchronized with the programming of the mounter.

Passive components, such as chip resistors or capacitors, are fed in vibratory feeders of either the bowl or linear type. 
Substrates in high production operations, such as the automotive field, permit automation. In most cases, this is not practical for the average hybrid shop because of the large amount of change-over time and because of the great number of different sizes that must be used. This problem can be facilitated if the hybrid manufacturer attempts to tool for a few sizes and tries to keep most of his production and tooling in these sizes of substrate. We would like to see a trend toward using the largest possible substrate, for example, $3 \frac{1}{2} \times 43 / 4$, and performing the die mounting, wire bonding, and other operations prior to scribing and breaking. This method is especially useful where hybrid technology is used to bond (die and wire) an array of identical carriers.

These design objectives are exemplified in the operator-controlled hybrid die mounter, shown schematically in Figure 4 (Plan View) and Figure 5 (Side Elevation). The machine provides for the various types of component presentation. All component presentation and substrates are on the nonmoving part of the machine. The optical train and the pick-up mechanism are part of the moving head. The substrates are silk screened prior to coming to the die bonding operation with systems of epoxies required.

The optical train is equipped with a programmable focusing device to insure that the die is always in focus at the CCTV Camera. The pick-up mechanism has a tube that moves in a $\mathbf{Z}$ direction and is also rotatable for theta correction. Below and in line with the pick-up axis, is the plunge-up tool. This is used for elevating the die onto the lowered bottom surface of the pick-up tool to avoid disturbing adjacent die and also to reduce the area of adhesive engagement between the bottom of the die and the top surface of the film. The aligned pick-up tool and plunger move as a unit in $\mathrm{X}$ and $\mathrm{Y}$.

The sequencing is pre-programmed and automatic. First, the head, with its optical referencing axis, is moved to a previously programmed alignment point on the substrate. If the point is not aligned with the crosshair on the TV monitor, the operator makes an $\mathrm{X}-\mathrm{Y}$ correction and enters this location. The head automatically moves to a second alignment and the sequence is repeated. If there is more than one substrate, the machine, through its pre-programmed sequence, permits the operator to make all necessary corrections by adjusting and entering two points per substrate.

Next, the head moves, for example, to one of the waffle packs. The micro-processor remembers the last site from which a die was removed and the head advances to the next pocket as part of the sequencing operation. If that pocket is empty for any reason, the operator presses an index button to cause the head to step over one pocket. If the first alignment site for the die is not on the crosshair, the operator, through the $\mathrm{X}-\mathrm{Y}$ control knobs, moves the head until the lower left bonding pad is over the crosshair. The operator then presses the "enter" button and the head automatically moves to the anticipated second alignment site on the die. Pressing the "enter" button after the second alignment causes the head to move until the pick-up axis is over the centroid of the die. The head picks up the die, rotates it through the amount of theta required to correct for both the error in die and substrate location, and places it onto the programmed bonding site on the substrate.

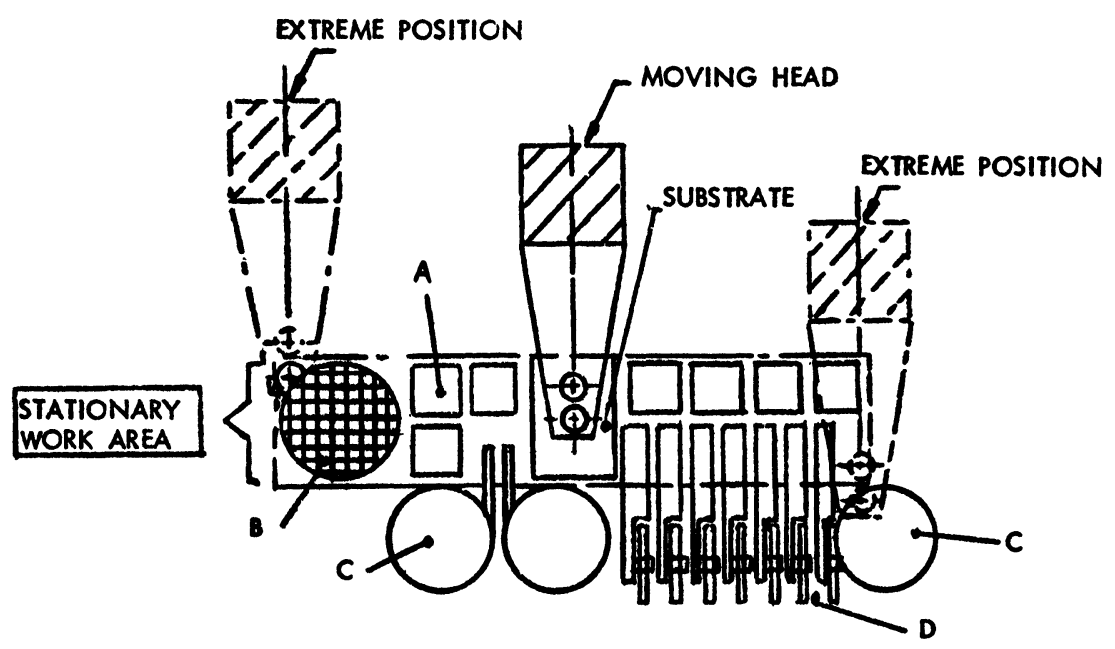

FIGURE 4 Schematic of an operator-controlled hybrid die mounter (plan view): a) waffle packs; b) wafer in matrix form; c) vibratory bowl feed of passive components; d) tape mounted die. 


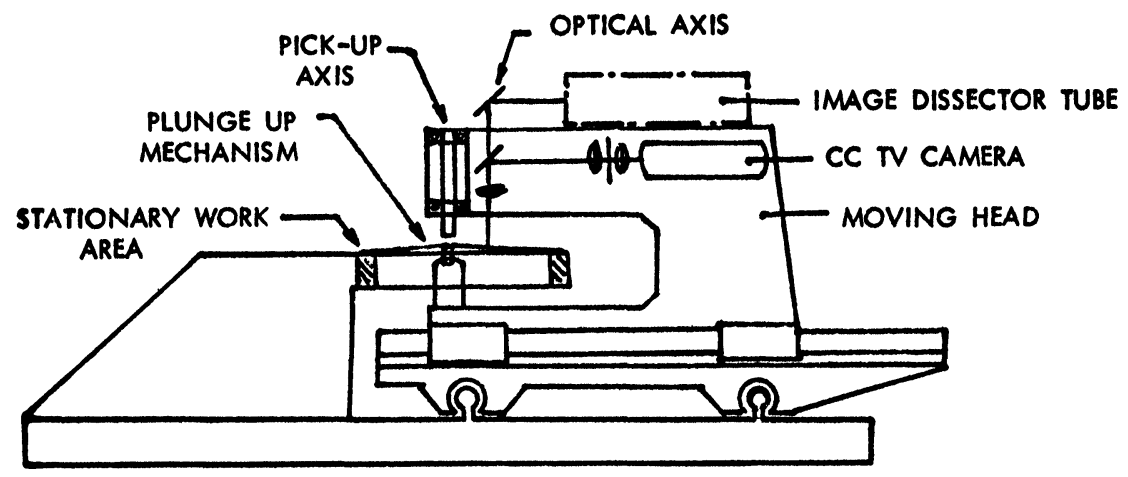

FIGURE 5 Schematic of an operator-controlled hybrid die mounter (side elevation) with automatic eye alignment (option).

Film mounted wafers, with or without plunge-up, are aligned in a manner similar to that of the waffle pack. Passive components are picked up normally without operator alignment, but parts out of tolerance can be re-aligned by switching to a manual alignment mode for operator override.

A more sophisticated machine system uses an automatic eye (see dotted line area in Figure 5) to perform the functions previously under operator control. The programming is similar to that for manual use, except the operator must select, in addition to the three points to establish orthogonality and the centre of the die, a signature with its centroid near the centre of the die. During automatic alignment, the optical axis is positioned over the anticipated centre of the signature. If the die is off $X, Y$, and theta, the optical axis is moved until it is at the centroid of the signature and the theta correction noted. The die is picked up at its mechanical centre and is rotated through the combined theta corrections. The eye also performs the additional function of by-passing any die which does not have a signature similar to that stored in memory for that specific die. This provides an automatic optical certification of the correctness of the die for the particular bonding site programmed.

Alignment using an operator is certainly a function of operator skill. We do not expect that the alignment time for die pick-up will require more than that used for wire bonding. Therefore, using 4 seconds to align a die, we feel that an overall cycle will take 6.6 seconds. This allows 300 milliseconds for each of pick-up and placing die and 1 second average for the head to travel from the pick-up position to the substrate and then to return to the pick-up position.

When using the automatic aligning system, the alignment time should be less than 1 second. Thus, on a similar basis, a total of 3.1 seconds is expected for a full align, pick-up and return to next pick-up position.

\section{REFERENCES}

1. M. H. Mehr and E. Mehr, Manual digital positioning in 2 axes; a comparison of joystick and track ball controls. Measurement Systems, Incorporated. Presented at the 16 th Annual Meeting of the Human Factors Society, October, 1972.

2. K\&S Model 1414 Automatic Wire Bonder with Automatic Alignment. Kulicke \& Soffa Industries, Inc., Horsham, PA, USA.

3. Hughes Hybrid Wire Bonders, Industrial Products Division, Carlsbad, CA, USA.

4. General Automation/Gaiser Tool Co., Santa Ana, CA, USA.

5. TAC TAPE, Mfg. by TAC, Woburn, MA, USA. 

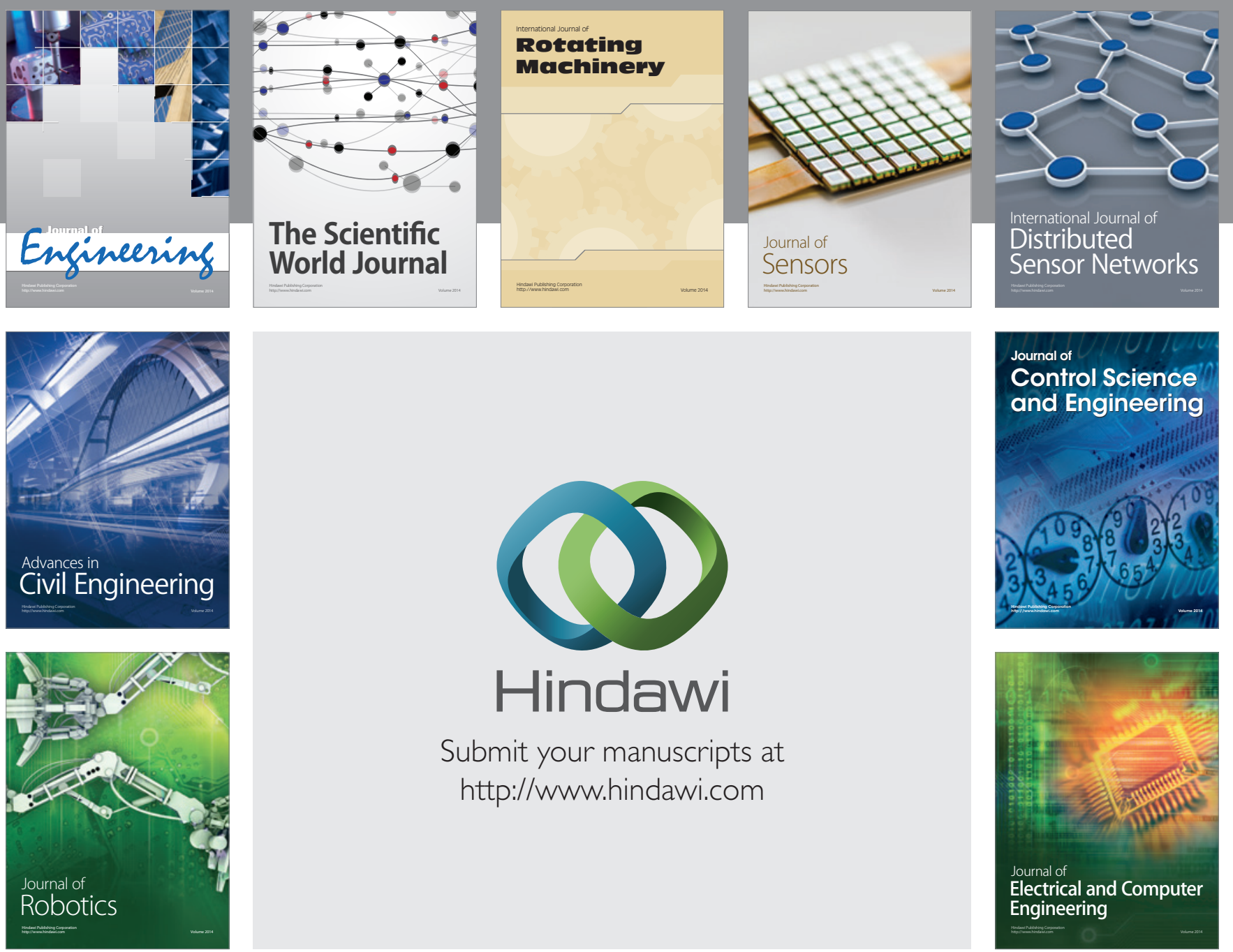

Submit your manuscripts at

http://www.hindawi.com
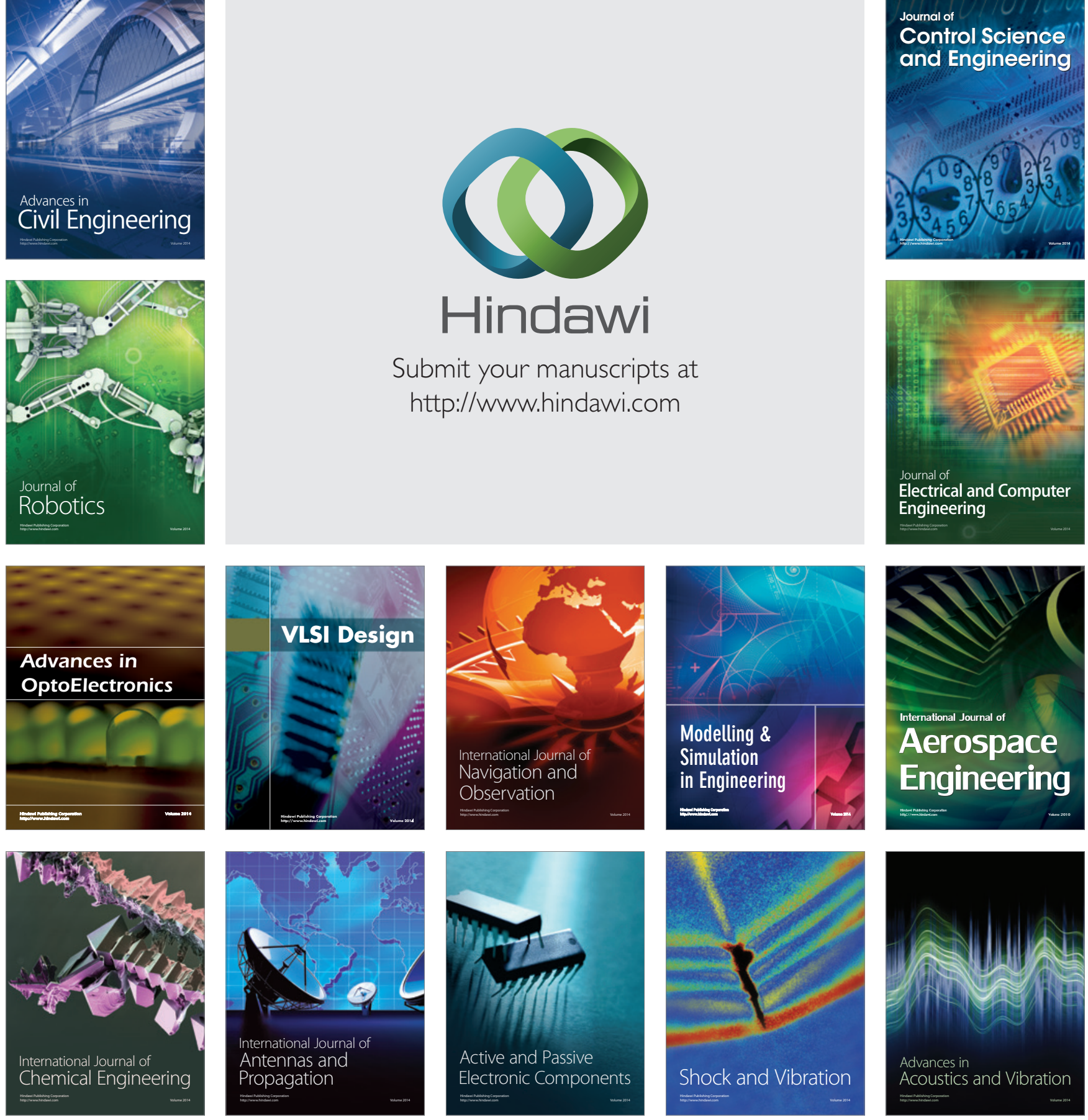